\title{
Velocity Estimation of the Main Portal Vein with Transverse Oscillation.
}

Brandt, Andreas Hjelm; Hansen, Kristoffer Lindskov; Nielsen, Michael Bachmann; Jensen, Jørgen Arendt

\section{Published in:}

Proceedings of 2015 IEEE International Ultrasonics Symposium

Link to article, DOI:

10.1109/ULTSYM.2015.0068

Publication date:

2015

Document Version

Peer reviewed version

Link back to DTU Orbit

Citation (APA):

Brandt, A. H., Hansen, K. L., Nielsen, M. B., \& Jensen, J. A. (2015). Velocity Estimation of the Main Portal Vein with Transverse Oscillation. In Proceedings of 2015 IEEE International Ultrasonics Symposium IEEE.

https://doi.org/10.1109/ULTSYM.2015.0068

\section{General rights}

Copyright and moral rights for the publications made accessible in the public portal are retained by the authors and/or other copyright owners and it is a condition of accessing publications that users recognise and abide by the legal requirements associated with these rights.

- Users may download and print one copy of any publication from the public portal for the purpose of private study or research.

- You may not further distribute the material or use it for any profit-making activity or commercial gain

- You may freely distribute the URL identifying the publication in the public portal 


\title{
Velocity Estimation of the Main Portal Vein with Transverse Oscillation
}

\author{
Andreas Hjelm Brandt ${ }^{\mathrm{a}, \mathrm{b}}$, Kristoffer Lindskov Hansen ${ }^{\mathrm{a}, \mathrm{b}}$, Michael Bachmann Nielsen ${ }^{\mathrm{a}}$, and Jørgen Arendt Jensen ${ }^{\mathrm{b}}$ \\ ${ }^{a}$ Dept. of Radiology, Copenhagen University Hospital, Denmark \\ ${ }^{\mathrm{b}}$ Center for Fast Ultrasound Imaging, Technical University of Denmark, Denmark
}

\begin{abstract}
This study evaluates if Transverse Oscillation (TO) can provide reliable and accurate peak velocity estimates of blood flow the main portal vein. TO was evaluated against the recommended and most widely used technique for portal flow estimation, Spectral Doppler Ultrasound (SDU). The main portal vein delivers blood from the bowls to the liver, and patients with certain liver diseases have decreased flow in the portal vein. Errors in velocity estimation with SDU are well described, when the beam-to-flow angle is $>70$ degrees. TO estimates the flow angle independently and is not limited by the beam-to-flow angle. It is less operators depended, as no angle correction is necessary. TO measurements were performed with a $3 \mathrm{MHz}$ convex probe (BK medical 8820e, Herlev, Denmark) connected to the experimental ultrasound scanner SARUS (Synthetic Aperture Real-time Ultrasound Scanner). SDU velocity measurements were performed with a commercial ultrasound scanner (BK 3000, BK Ultrasound, Herlev Denmark) and a convex probe (BK ultrasound 6C2, Herlev, Denmark). Ten healthy volunteers were scanned, and recordings of the portal flow during 3-5 heartbeats were conducted with an intercostal and subcostal view. Intercostal TO peak velocities were not significantly different from SDU peak velocities $(\mathrm{TO}=0.203 \mathrm{~m} / \mathrm{s}, \mathrm{SDU}=0.202 \mathrm{~m} / \mathrm{s}, \mathrm{p}=0.94)$. Subcostal and Intercostal obtained TO values were not significantly different (intercostal mean $T O=0.203 \mathrm{~m} / \mathrm{s}$, subcostal mean $T O=0.180 \mathrm{~m} / \mathrm{s}, \mathrm{p}=0.26$ ). SDU values obtained intercostal and subcostal were significantly different (intercostal mean SDU $=0.202 \mathrm{~m} / \mathrm{s}$, subcostal mean SDU $=0.320 \mathrm{~m} / \mathrm{s}, \quad p<0.001$ ). Standard deviation for TO beam-to-flow angle was $10.3^{\circ}-91.5^{\circ}$, indicating a large beam-to-flow angle variability in the portal vein. This can affect the peak velocity estimation, and is not addressed in SDU. The TO convex array implementation provides the first vector velocity measurements below $60 \mathrm{~mm}$ (mean 89mm), and is a useful alternative for flow estimation in abdominal ultrasound. It may provide new information of abdominal fluid dynamics and yield both velocity and angle estimates for a more realistic flow characterization.
\end{abstract}

Keywords - Transverve Oscillation, Spectral Doppler, Vector Velocity Estimation, Main Portal Vein, Ultrasound Velocity Estimation

\section{INTRODUCTION}

The hepatic portal vein conducts blood from the gastrointestinal tract and spleen to the liver. Increased blood pressure in the portal vein is denoted portal hypertension and a is major complication in liver diseases, most commonly liver cirrhosis. Complications to portal hypertension (e.g. ascites, esophageal and gastric varices, and splenomegaly) are often fatal [1]. Portal vein hypertension can lead to a reduced velocity and in advanced stages the portal vein flow becomes reversed [2, 3]. Ultrasound is usually the first diagnostic modality for assessment of portal flow, since it is easily accessible, radiation free, and cheap. The European Federation of Societies for Ultrasound in Medicine and Biology recommends evaluation of peak velocity in the main portal vein in patients suspected for portal hypertension using Spectral Doppler Ultrasound (SDU) [4].

Errors in velocity measurement with SDU are well described, when the beam-to-flow angle is $>70$ degrees to the main portal vein [5]. Furthermore, operator dependency has shown to be a major bias in Doppler ultrasound [6]. Other factors as body position, phase of respiration, timing of meals, exercise and cardiac output can affect the accuracy of velocity measurements, and change the flow profile of the liver vasculature. However, Doppler ultrasound is accepted as a useful technique for evaluating patients with cirrhosis and portal hypertension [7].

Transverse Oscillation (TO) measures the vector velocity independently of the beam-to-flow angle. Both the axial and the transverse velocity are found and used to calculate the vector velocity. The axial velocity is found using conventional velocity estimation, while the transverse velocity is found by manipulating the receive beamforming. TO is described thoroughly by Jensen, Munk and Udesen [8, 9, 10]. Vector velocity is a novel technique, and recent studies have indicated that TO is ready for clinical scanning [11-15]. Clinical TO examinations have until now been limited by the implementation, as TO only worked on a linear array transducer with a scan depth of approximately $60 \mathrm{~mm}$. For abdominal scanning an increased scan depth is needed. TO was implemented on a convex array transducer for this purpose [16].

The preferred scan position for evaluation flow in the main portal vein is an intercostal view (between the ribs on the right side of the thorax) (Fig. 1). The beam-to-flow angle is in this position around 0 degree, as the vessel flow is going straight towards the transducer, and SDU velocity estimation in this position is reliable [4]. With a subcostal view (under the ribs curvature) (Fig. 1), the main portal vein can be visualized, but the beam-to-flow angle is in this position about 90 degrees, thus, impossible to measure with SDU. TO may provide reliable velocity estimation with this view. The hypothesis of this study is therefore, firstly to determine if TO on a convex 
array can provide reliable peak velocity estimates in the main portal vein with an intercostal view compared to SDU, and secondly, to determine if TO can provide an accurate flow estimate of the portal vein with a subcostal view.

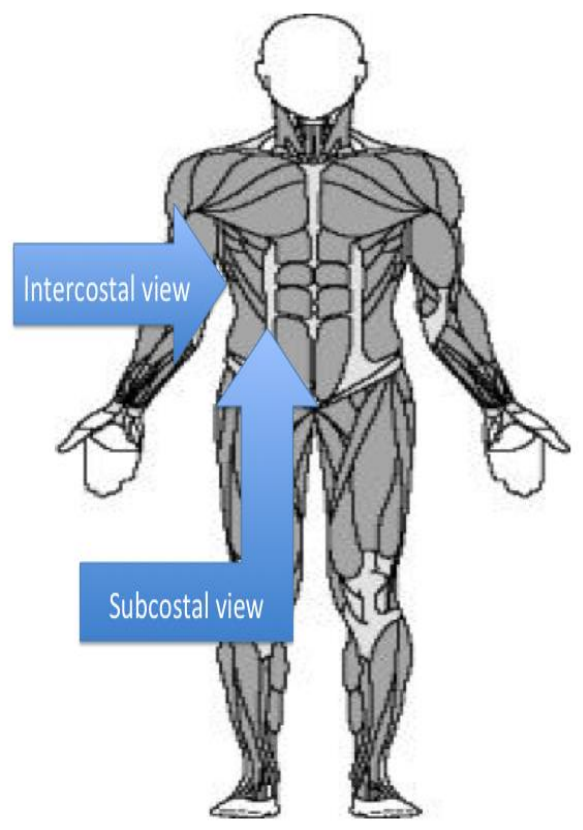

Figure 1: Schematic illustration of the intercostal and subcostal scan position.

\section{MATERIALS AND METHODS}

\section{A. Equipment and data acquisition}

TO velocity measurements were performed with a $3 \mathrm{MHz}$ convex probe (BK medical 8820e, Herlev, Denmark) connected to the experimental ultrasound scanner SARUS (Synthetic Aperture Real-time Ultrasound Scanner). SDU velocity measurements were performed with a commercial ultrasound scanner (BK 3000, BK Ultrasound, Herlev Denmark) and a convex probe (BK Ultrasound 6C2, Herlev, Denmark). An in-house developed algorithm in MATLAB (MathWorks, Natick, MA, USA) was used for peak velocity estimation with TO and is previously described thoroughly by Jensen et al. [16]. Beam-to-flow angle for TO was calculated by the vector diversity and averaged along the centerline of the vessel. Standard deviation for the beam-to-flow angle specified vector diversity for the mean beam-to-flow angle of TO. SDU peak velocities and fixed beam-to-flow angle information was gathered with the standard spectral Doppler setup on the commercial scanner.

\section{B. Patiens and Scan Setup}

Ten male healthy volunteers (mean age: 28.8 ; range: $26-32$ ) were included in the study. Health status of each volunteer was obtained by interview. All were included after informed consent and after obtained approval by the Danish National Committee on Biomedical Research Ethics (H-1-2014-FSP072). All volunteers were scanned in supine position by a single medical doctor (A.H.B.). The volunteers were asked to hold their breath in a mid or full respiratory level, while measurements were performed. Blood pressure and heart rate were assessed before the scan. With both methods, the portal flow was estimated in a intercostal and subcostal scan position, corresponding to 4 measurements for each volunteer. The commercial scanner was used for guidance before scanning with the SARUS, as the preview image for SARUS had limited frame rate and image quality. Each participant was scanned with both techniques within $30 \mathrm{~min}$.

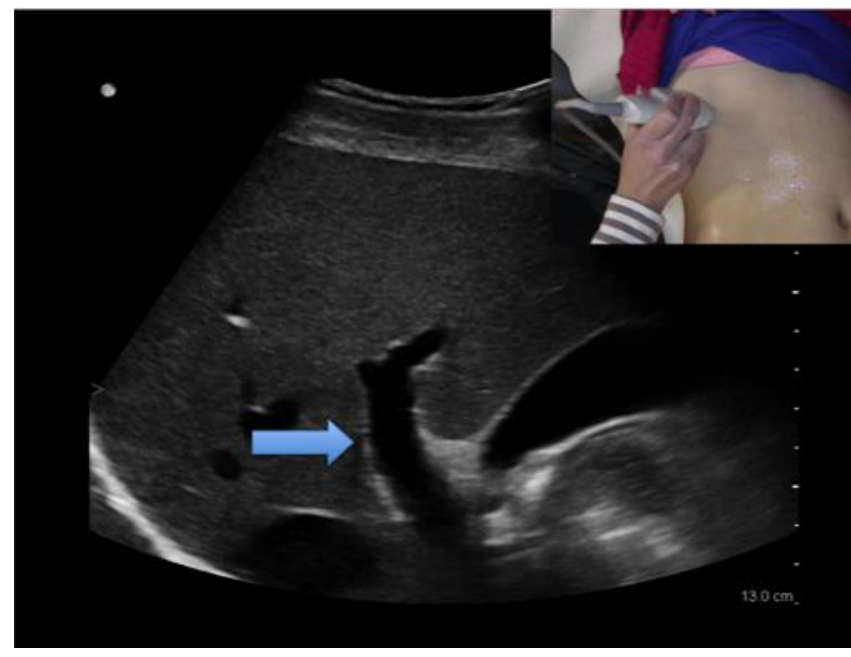

Figure 2: Intercostal view of the main portal vein (blue arrow) scanned with the commercial scanner.

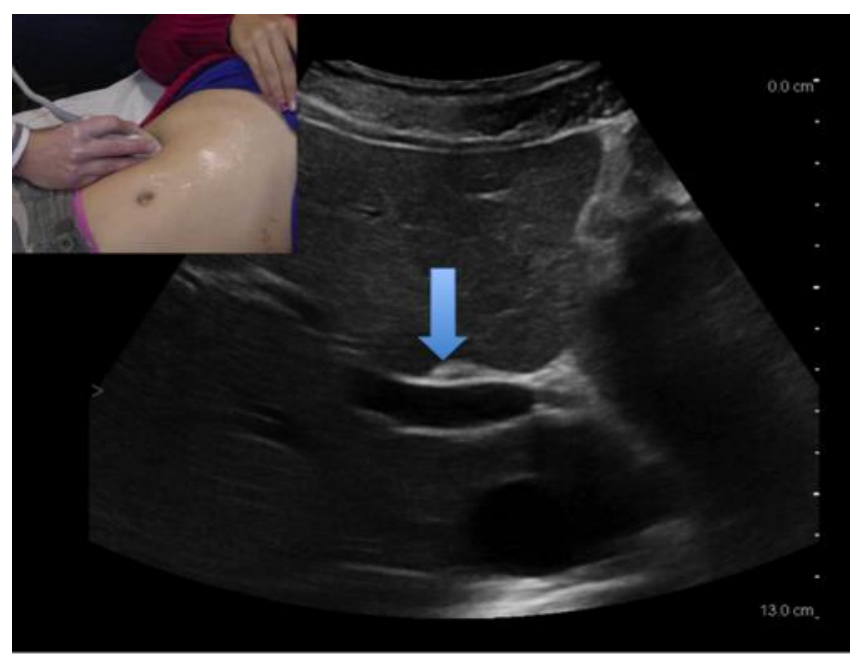

Figure 3: Subcostal view of the main portal vein (blue arrow) scanned with the commercial scanner.

\section{Statistics}

For descriptive analyses, the mean peak velocity and standard deviation were calculated for subcostal and intercostal scan position with TO and SDU. Differences between SDU and TO was analyzed with a paired t-test for both views. As the peak velocities values of the subcostal and intercostal view should be the same regardless of the method employed, the values were used as their own reference and 
differences for each method was analyzed with a paired t-test. Statistical significance level was set at 0.05. Mean beam-toflow angle for TO was calculated and given in mean for intercostal and subcostal view with standard deviation presenting vector diversity. Data were handled in with MATLAB (MathWorks, Natick, MA, USA) and Microsoft Excel (Redmond, WA, USA).

\section{RESULTS}

All participants had a normal heart rate (mean: 61 beats/min (range: 47-87) and blood pressure (mean systolic pressure 127 (range: 108-140); mean diastolic pressure 76 (range: 74-85)). BMI values were (mean BMI 23.9; range: 20.9-27.2).

Peak velocities from all volunteers for TO and SDU are shown in Figure 4. Intercostal obtained TO peak velocities were not significantly different from intercostal obtained SDU peak velocities (mean $\mathrm{TO}=0.203 \mathrm{~m} / \mathrm{s}$, mean $\mathrm{SDU}=0.202 \mathrm{~m} / \mathrm{s}$, $p=0.94)$. Subcostal obtained TO values were significantly different from subcostal obtained SDU values (mean $\mathrm{TO}=0.180 \mathrm{~m} / \mathrm{s}$, mean $\mathrm{SDU}=0.320 \mathrm{~m} / \mathrm{s}, \quad p<0.001)$. TO peak velocity obtained with an intercostal view were not significantly different from TO obtained values with a subcostal view (intercostal mean $\mathrm{TO}=0.203 \mathrm{~m} / \mathrm{s}$, subcostal mean $\mathrm{TO}=0.180 \mathrm{~m} / \mathrm{s}, p=0.26)$. Intercostal obtained SDU values were significantly different from subcostal obtained values (intercostal mean $\mathrm{SDU}=0.202 \mathrm{~m} / \mathrm{s}$, subcostal mean $\mathrm{SDU}=0.320 \mathrm{~m} / \mathrm{s}, p<0.001)$.

Range for the TO mean beam-to-flow angle was $9^{\circ}-61^{\circ}$ with the intercostal view and $4^{\circ}-39^{\circ}$ with SDU. The mean TO subcostal view beam-to-flow angle range was $53^{\circ}-130^{\circ}$ and $57^{\circ}-99^{\circ}$ with SDU. Standard deviation for TO mean beam-toflow angle was between $7.8^{\circ}-91.5^{\circ}$. Mean scan depth for TO was $89 \mathrm{~mm}$ with a range of $59-104 \mathrm{~mm}$. An example from a volunteer scanned in intercostal view with SDU and TO is shown in Figs. 5 and 6.

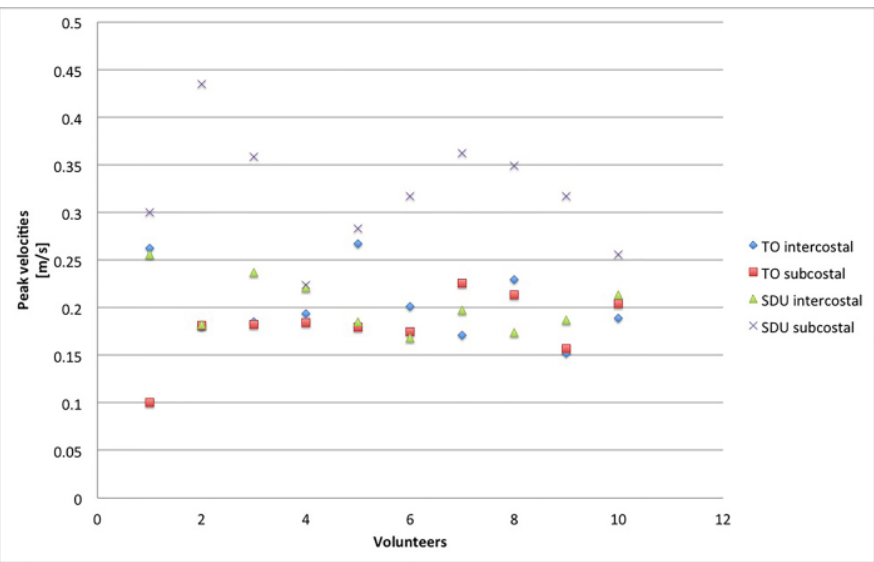

Figure 4: Peak velocities for each volunteer and each view obtained with both techniques.

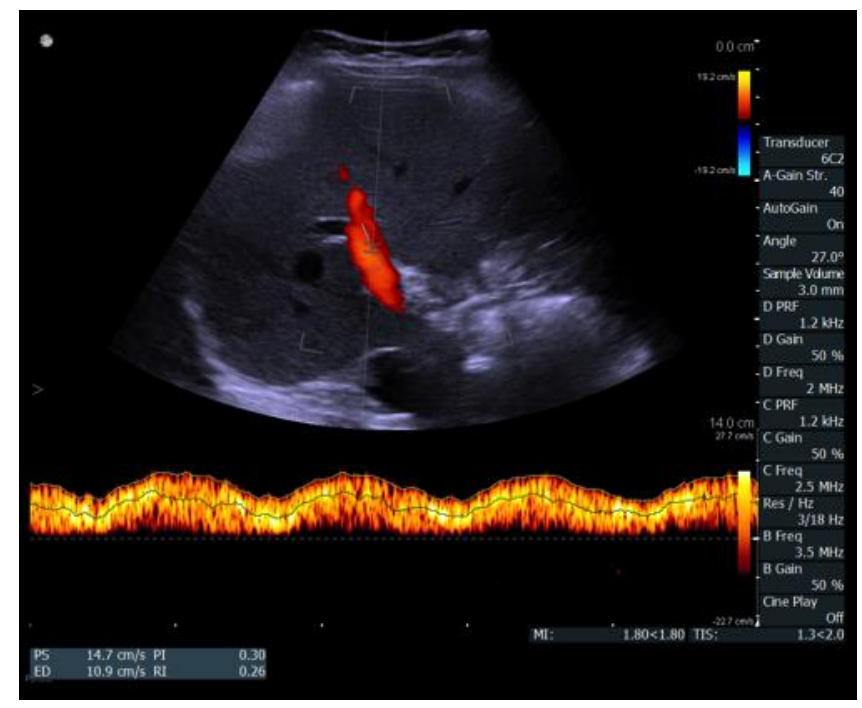

Figure 5: Example from volunteer scanned with the commercial scanner with a intercostal view.

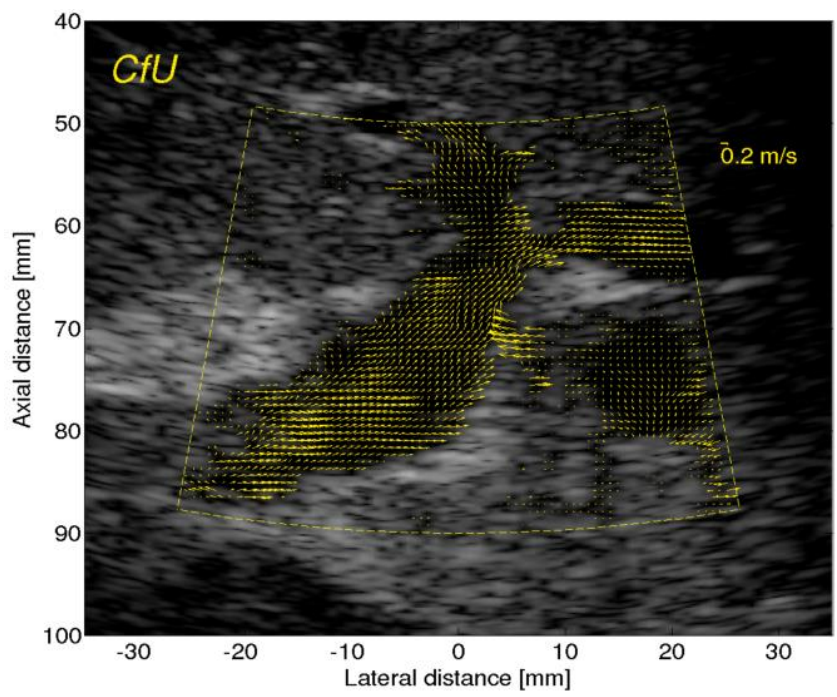

Figure 6: Example from volunteer with the TO setup from a intercostal view.

\section{DISCUSSION}

This is the first study to examine peak velocity obtained by TO in the main portal compared to SDU. SDU is only reported to measure correct peak velocities with an intercostal view [4], and overestimates the flow with a subcostal view. With the accepted view for SDU (intercostal), TO peak velocities were not significantly different from SDU peak velocities, while values were as expected significantly different with the subcostal view. Measurement performed with a subcostal and intercostal view for TO were not significantly different, while values were significantly different for SDU. This clearly shows TO angle independence, and that TO can offer a reliable evaluation of the peak velocity in the portal vein with views, which are not achievable with SDU. For clinical evaluation of patients treated with liver surgery or liver transplantation, this could be an advantage since the liver 
anatomy and portal vein position is altered, and hereby hampering the evaluation with SDU [4].

Performing SDU measurement requires training. Adjusting the Doppler angle, position the sample volume, adjusting of the spectral gain, and adjustment of the display scale are all known sources of error [5, 17]. Experienced ultrasound users are known to estimate errors up to $28 \%$ in peak velocity values on vessel phantoms [6]. The solution to SDU errors could be TO, as angle independent vector velocity estimation with TO is less operator dependent than SDU $[12,18]$.

Standard deviation for the beam-to-flow angle of TO has previously been reported to be around $3^{\circ}$ in a simulation phantom study [8], but the measured standard deviation in this study was $10.3^{\circ}-91.5^{\circ}$. There is, thus, large vector diversity, when measuring flow in the portal vein, indicating that the flow is not laminar. This greatly affects the peak velocity estimate and cannot be detected with SDU, since SDU assumes a fixed beam-to-flow angle. TO may therefore provide new information of abdominal fluid dynamics and yield both velocity and angle estimates for a more realistic flow characterization.

Furthermore, this is the first TO convex array implementation providing vector velocity measurements below $60 \mathrm{~mm}$. This shows that TO is a useful angle independent alternative for flow estimation in medical abdominal ultrasound.

Future studies where the reproducibility of TO compared to SDU in a larger study population are under preparation. A TO implementation on a commercial scanner is considered for a larger study with patients suffering from liver disease.

\section{CONCLUSION}

This study indicates that TO is a useful alternative for velocity estimation in the main portal vein. TO estimated the same peak velocities as SDU in healthy volunteers. Furthermore, TO estimates identical values with a subcostal view, which is inapplicable for SDU, thus, a new insonation window of the portal flow is introduced with TO. TO can provide new information to abdominal fluid dynamics. This could improve the clinical examination of patients suffering from liver disease.

\section{ACKNOWLEDGMENT}

The authors wish to thank all participating volunteers. The study was supported by grant number 82-2012-4 from The Danish National Advanced Technology Foundation and by BK Ultrasound.

\section{REFERENCES}

[1] N. Dib, F. Oberti, and P. Cales, "Current management of the complications of portal hypertension: variceal bleeding and ascites," CMAJ, vol. 17 no.10, pp.1433-1443, 2006.

[2] M. Davis and W.K. Chong, "Doppler Ultrasound of the Liver, Portal Hypertension, and Transjugular Intrahepatic Portosystemic Shunts," Ultrasound Clinics, vol. 9 no.4 pp. 587-604, 2014.

[3] T. Kok, E.J. van der Jagt, E.B. Haagsma, C.M. Bijleveld, P.L. Jansen, and W.J. Boeve, "The value of Doppler ultrasound in cirrhosis and portal hypertension," Scand J Gastroenterol Suppl, vol. 230 pp. 82-88, 1999.

[4] A. Berzigotti and F. Piscaglia, "Ultrasound in portal hypertension--part 2--and EFSUMB recommendations for the performance and reporting of ultrasound examinations in portal hypertension," Ultraschall Med vol. 33no.1 pp. 8-32, 2006.

[5] S. F. Stewart, "Effects of transducer, velocity, Doppler angle, and instrument settings on the accuracy of color Doppler ultrasound," Ultrasound Med Bio, vol. 27 no.4 pp. 551-564, 2001.

[6] E.Y.L. Lui, A.H. Steinman, R.S.C. Cobbold, and K.W. Johnston, "Human factors as a source of error in peak Doppler velocity measurement," J Vasc Surg, vol. 42 no.5 pp. 972-979, 2005.

[7] J.B. Kruskal, P.A. Newman, L.G. Sammons, and R.A. Kane, "Optimizing Doppler and color flow US: application to hepatic sonography, " Radiographics vol. 24 no.3 pp. 657-675, 2004.

[8] J.A. Jensen and P. Munk, "A new method for estimation of velocity vectors," IEEE Trans Ultrason Ferroelectr Freq Control, vol.45, no.3, pp837-851, 1998.

[9] J.A. Jensen, “A new estimator for vector velocity estimation," IEEE Trans Ultrason Ferroelectr Freq Control, vol. 48 no.4, pp. 886-894, 2001.

[10] J. Udesen and J.A. Jensen, "Investigation of transverse oscillation method," IEEE Trans Ultrason Ferroelectr Freq Control, vol. 53 no.5, pp. 959-971, 2006.

[11] P. Tortoli, M. Lenge, D. Righi, G. Ciuti, H. Liebgott, and S. Ricci, "Comparison of carotid artery blood velocity measurements by vector and standard Doppler approaches," Ultrasound Med Bio, vol. 41 no.5 pp. 1354-1362, 2015.

[12] M.M. Pedersen, M.J. Pihl, P. Haugaard, J.M. Hansen, K.L. Hansen, M.B. Nielsen, and J.A. Jensen, "Comparison of real-time in vivo spectral and vector velocity estimation," Ultrasound Med Bio, vol. 38 no.1 pp. 145-151, 2012.

[13] P.M. Hansen, J.B. Olesen, M.J. Pihl, T. Lange, S. Heerwagen, M.M. Pedersen, M. Rix, L. Lönn, J.A. Jensen, and M.B. Nielsen, "Volume flow in arteriovenous fistulas using vector velocity ultrasound," Ultrasound Med Bio, vol. 40 no.11, pp. 2707-2714, 2014.

[14] K.L. Hansen, H. Møller-Sørensen, M.M. Pedersen, P.M. Hansen, J. Kjaergaard, J.T. Lund, J.C. Nilsson, J.A. Jensen, and M.B. Nielsen, "First report on intraoperative vector flow imaging of the heart among patients with healthy and diseased aortic valves," Ultrasonics, vol. 56 pp. 243-250, 2014.

[15] K.L. Hansen, M.M. Pedersen, H. Møller-Sørensen, J. Kjaergaard, J.C. Nilsson, J.T. Lund, J.A. Jensen, and M.B. Nielsen, "Intraoperative cardiac ultrasound examination using vector flow imaging," Ultrason Imaging, vol. 25 no.4 pp. 318-332, 2013.

[16] J.A. Jensen, A.H. Brandt, and M.B. Nielsen, "In-vivo convex array vector flow imaging," Proc IEEE Ultrason Symp, pp. 333-336, 2014.

[17] T.L. Szabo, ”Diagnostic ultrasound imaging: Inside out," Elsevier, 2014.

[18] P.M. Hansen, M.M. Pedersen, K.L. Hansen, M.B. Nielsen, and J.A. Jensen, "New technology - demonstration of a vector velocity technique," Ultraschall, vol. 32 no. 2 pp. 213-215, 2011. 